\title{
Evaluation des postgradualen Studiengangs „Master of Science (M. Sc.) in Addiction Prevention and Treatment" - Suchthilfe als Studiengang zur Verbesserung der therapeutischen und wissenschaftlichen Kompetenz von Suchthilfemitarbeitern
}

\section{Michael Klein \\ Tanja Hoff}

Evaluation of the Postgraduate Course of Studies „Master of Science (M. Sc.) in Addiction Prevention and Treatment" - Addiction Care as a Course of Studies for the Improvement of the Therapeutic and Scientific Competence of Employees in the Addiction Care

\section{Zusammenfassung}

Der Masterstudiengang „Master of Science (M. Sc.) in Addiction Prevention and Treatment" stellt das erste Weiterbildungsstudium in Deutschland dar, das innerhalb von vier Semestern eine berufliche Weiterbildung in der Suchthilfe mit einem europaweit anerkannten, postgradualen Hochschulstudium verbindet und darüber hinaus Absolventen des Studienschwerpunkts Suchttherapie zu VDR-anerkannten Suchttherapeuten qualifiziert. Die Semester-, Seminar- und Abschlussevaluationen des ersten Weiterbildungskurses bei 24 Teilnehmern belegen eine hohe Qualität des Studiums hinsichtlich der vermittelten wissenschaftlichen und therapeutischen Kompetenzen im Bereich Suchttheorie, Suchttherapie und berufliche Selbstreflexion. Verbesserungsbedarf zeigt sich im organisatorischen Aufbau und der Transparenz von Lehrveranstaltungen sowie von Leistungsanforderungen und in der Vermittlung von Qualitätskontrolle und -sicherung in der Therapie. 53,8\% der Teilnehmer konnten während der Weiterbildung ihre berufliche Position halten, $38,5 \%$ verbessern.

\section{Schlliisselwörter}

Suchthilfe · Suchttherapie · Suchtprävention · Evaluation · Weiterbildung $\cdot$ postgradualer Masterstudiengang

\section{Abstract}

The master course of studies "Master of Science (M. Sc.) in Addiction Prevention and Treatment" represents the first postgraduate course of studies in Germany that combines within four termtimes a postgraduate professional education in addiction help with an all over Europe recognized postgraduate university study. The evaluation of the term-times, of the seminars and the final evaluation of the first postgraduate course with 24 participants prove a high quality of the study with regard to the taught scientific and therapeutic competences in the field of addiction theory, addiction therapy and professional self reflexion. There still is need, however, of improvement in the organizational structure and in the transparency of the courses, of the achievment requirements and in teaching of quality control and quality assurance in the therapy. $53.8 \%$ of the participants could hold their occupational position during the course, $38.5 \%$ were able to improve it.

Key words

Addiction care · drug and alcohol therapy · addiction prevention . evaluation $\cdot$ professional training $\cdot$ postgraduate course

\section{Einleitung}

Im Bereich der Suchttherapie und zunehmend auch im Bereich der Suchtprävention ist seit einigen Jahren ein kontinuierlicher Bedarf an qualifiziertem hochprofessionellem Personal zu ver- zeichnen. Weiterbildungen im Bereich der Suchthilfe, speziell der Suchttherapie, weisen meist jedoch keinen engen Bezug zur aktuellen wissenschaftlichen Forschung auf. Die Verbindung zwischen den suchttherapeutischen Weiterbildungsprogrammen einerseits und den Fortschritten in Wissenschaft und For-

Prof. Dr. Michael Klein · Katholische Fachhochschule NW, Abt. Köln, Forschungsschwerpunkt Sucht . Wörthstr. 10·50668 Köln·E-mail: mikle@t-online.de 
schung andererseits ist aber in höchstem Maße notwendig, um auch in Zukunft die Qualität der Suchthilfe sicherzustellen. Erst seit der Novellierung des Hochschulrahmengesetzes im Jahre 1998 hat eine verstärkte Verwissenschaftlichung der Weiterbildungen durch die Hochschulen begonnen. Im Folgenden werden die Entwicklung und Umsetzung eines Curriculums dargestellt, das die Erfordernisse der beruflichen Weiterbildung mit den Möglichkeiten eines postgradualen Hochschulstudiums verbindet: der „Masterstudiengang Suchthilfe“ („Master of Science [M. Sc.] in Addiction Prevention and Treatment") an der Katholischen Fachhochschule Nordrhein-Westfalen, Abteilung Köln. Dies ist der erste akademische Studiengang, der Suchthilfe für Absolventen aus den Bereichen Psychologie, Medizin und soziale Arbeit studierbar macht. Im Rahmen des Studienmodells werden die beiden Haupttätigkeitsmerkmale in der Suchthilfe - Suchtprävention und Suchttherapie - in einem berufsbegleitenden viersemestrigen Studiengang praxisnah und wissenschaftlich fundiert gelehrt. Für Studierende mit dem Schwerpunkt Suchttherapie ist ein vom Verband Deutscher Rentenversicherungsträger (VDR) anerkannter Weiterbildungsgang in verhaltenstherapeutisch orientierter Suchttherapie in den Studiengang integriert. Der Studiengang wird derzeit auf die hochschulrechtliche Akkreditierung vorbereitet, welche für 2004 vorgesehen ist. Im vorliegenden Beitrag werden die Ergebnisse der Seminar-, Semester- und Abschlussevaluation des ersten Masterkurses und die Weiterentwicklung und Zukunftschancen des Studiengangs dargestellt.

\section{Inhalte und Struktur des postgradualen Studiengangs „Master of Science (M. Sc.) in Addiction Prevention and Treatment“}

Träger des postgradualen Masterstudiengangs „Suchthilfe“ mit der integrierten berufsbegleitenden Weiterbildung „Suchttherapeut/in“ (VDR-anerkannt) ist die Katholische Fachhochschule NW. Der Studiengang wurde in Kooperation mit dem Sozialdienst Katholischer Männer e.V. Köln (Drogenhilfe) und dem Diözesan-Caritasverband Köln (Suchthilfe) entwickelt. Das Masterstudium wird abgeschlossen mit dem akademischen Titel „Master of Science (M. Sc.) in Addiction Prevention and Treatment" (europaweit anerkannt) und für den Studienschwerpunkt „Suchttherapie“ zusätzlich mit dem Zertifikat „Suchttherapeut/ in KFH NW“ (VDR-anerkannt). Die Dauer des berufsbegleitenden Studiums beträgt vier Semester; das Studium endet mit einer Masterthesis und einem Kolloquium. Die Größe der Studiengruppe, die von zwei erfahrenen Supervisoren nach dem Kursleitersystem begleitet wird, beträgt 24 Teilnehmer; während der Supervisionen und Selbstreflexionen werden zwei Parallelgruppen à 12 Teilnehmer gebildet. Der Studiengang wird an Wochenenden (10 bis 12 Wochenenden pro Semester) und darüber hinaus an 3 bis 4 Blockwochen während des gesamten Studiengangs durchgeführt. Die gesamte durch Dozenten und Supervisoren geleitete Weiterbildungszeit beträgt $812 \mathrm{Std}$. à 45 Minuten; hinzu kommen Eigenstudium und Intervision. Das Curriculum wird von Therapeuten und Supervisoren durchgeführt, die über umfassende Erfahrung in der Behandlung von Suchterkrankungen verfügen.
Abb. 1 stellt die Aufteilung der Studieninhalte dar. Das Grundlagenstudium ist unterteilt in Suchtpsychologie, Suchtmedizin, soziale Suchtarbeit, Recht und Interdisziplinäre Suchtforschung. Der Studiengang gliedert sich sodann in die beiden Schwerpunkte Suchtprävention und Suchttherapie, von denen zu Beginn des Studiums ein Schwerpunkt von den Studierenden verbindlich gewählt werden muss. Der suchttherapeutische Schwerpunkt ist verhaltenstherapeutisch orientiert mit Akzent auf kognitiven und systemischen Methoden. Die praktischen Lehrelemente umfassen Techniken beruflichen Handelns (z.B. Therapiemethoden), Supervision und berufliche Selbstreflexion.

Aufnahmekriterien für den Studiengang sind innerhalb des Studienschwerpunktes Suchttherapie ein Hochschulabschluss als Arzt, Dipl.-Psychologe, Dipl.-Sozialarbeiter (FH) oder Dipl.-Sozialpädagoge (FH). Für den Schwerpunkt Suchtprävention werden darüber hinaus die meisten Studienabschlüsse in den Humanberufen, z.B. Dipl.-Soziologe, Dipl.-Pädagoge, Lehrer, Religionspädagogen u. ä. zugelassen. Bei nichtdeutschen, aber europäischen Abschlüssen sind Äquivalenzbescheinigungen, bei nicht europäischen $\mathrm{Ab}$ schlüssen ist eine genaue Auflistung der besuchten Veranstaltungen erforderlich. Die Teilnehmer müssen mindestens als Halbtagskraft (= 19,25 Std./Woche) in einer Einrichtung der Suchttherapie (z.B. Entgiftung, Entwöhnung, Beratung), der Suchtprävention (z.B. auch Schule) oder in einem benachbarten Arbeitsfeld mit Suchtkranken oder -gefährdeten (z.B. Wohnungslosenhilfe, Jugendhilfe, Bewährungshilfe u. ä.) tätig sein.

\section{Soziodemografische Daten der Teilnehmer des ersten Masterstudiengangs}

Der erste Kurs des „Masterstudiengangs Suchthilfe“ mit 24 Teilnehmern begann im Frühjahr 2001, danach folgte jährlich ein neuer Masterkurs mit jeweils 24 Teilnehmern. Im Frühjahr 2004 beginnt der vierte Studienkurs. Gegenstand dieser Evaluation sind die Beurteilungen der Teilnehmer des ersten Masterkurses hinsichtlich der Qualität der Weiterbildung. Am ersten Masterstudiengang nahmen 16 Frauen und acht Männer teil (Tab.1). Hinsichtlich des Hochschulabschlusses sind mehrheitlich zu

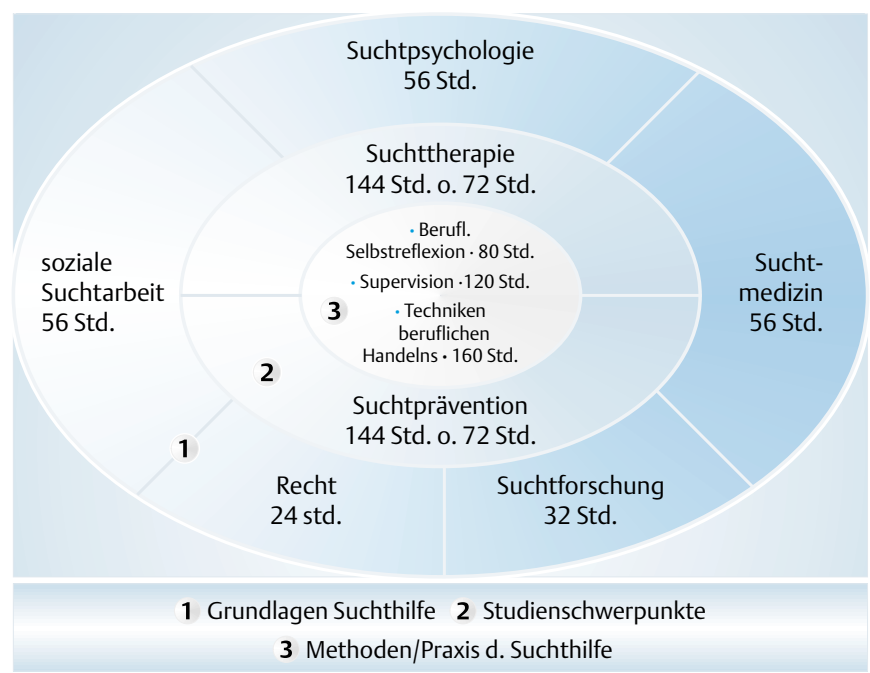

Abb. 1 Masterstudiengang Suchthilfe - Aufteilung der Studieninhalte 
Tab. 1 Demographische Daten der Teilnehmer des ersten Masterkurses

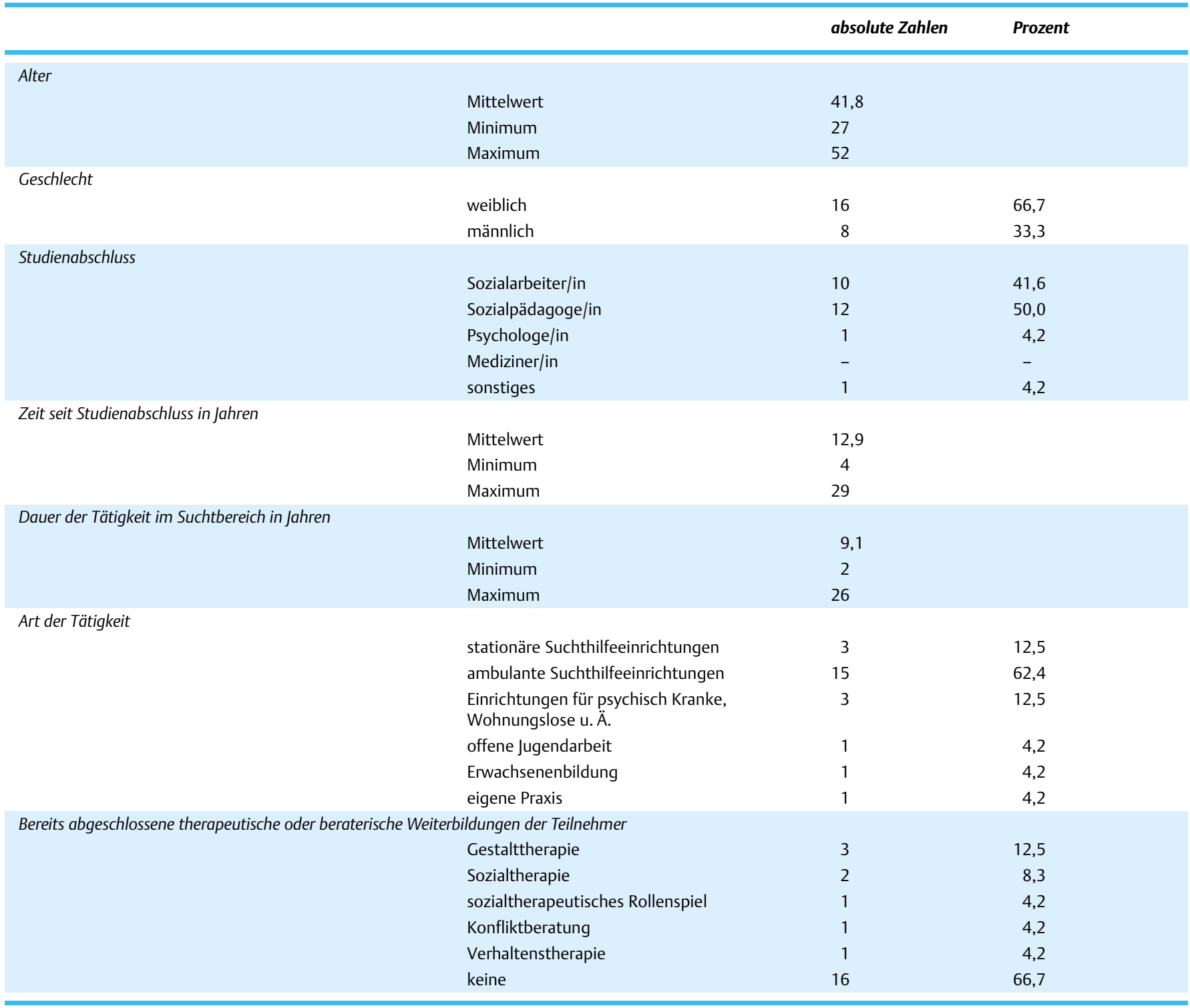

gleichen Anteilen Sozialarbeiter und Sozialpädagogen vertreten. Die Teilnehmer weisen mit einer durchschnittlichen Tätigkeitsdauer von 9,1 Jahren in suchtrelevanten Arbeitsbereichen bereits ausgeprägte Erfahrungen mit Suchtpatienten auf; mit 62,4\% arbeitet der überwiegende Teil der Masterstudierenden in ambulanten Suchteinrichtungen. Zum derzeitigen Zeitpunkt haben alle Teilnehmer dieses Kurses die erforderlichen Seminare des Masterstudiengangs abgeschlossen; sechs Teilnehmer haben die Weiterbildung mit der eingereichten und anerkannten Masterthesis endgültig beendet; kein Teilnehmer hat das Studium vorzeitig abgebrochen.

\section{Methodik der Evaluation}

Die Teilnehmer wurden sowohl nach jedem Seminar als auch nach jedem Semester zu ihrer persönlichen Einschätzung der Qualität der Lehrveranstaltungen befragt. In den Seminarevaluationen wurden Bewertungen auf einer sechsstufigen Skala erho- ben zu: der Qualität der Lerninhalte, der beruflichen Relevanz des Gelernten für die eigene berufliche Tätigkeit in der Suchthilfe, der Qualität des fachlichen Austauschs mit den anderen Teilnehmern, dem Dozenten und der Qualität seiner Lehrinhalte sowie -methodik, der Struktur und Dokumentation, dem Schwierigkeitsgrad, Lehrumfang, Lehrklima und der Gesamtbewertung des Seminars. Zusätzlich wurden die Teilnehmer um eine Einschätzung ihres eigenen Befindens gebeten und konnten qualitative Angaben zu kritischen, aber auch besonders positiven Aspekten des Seminars machen. In den Semesterevaluationen wurden ebenfalls auf einer sechsstufigen Skala Beurteilungen der Teilnehmer erhoben und zwar zu: der Koordination der Lehrangebote, der Verwaltung, Organisation, dem Service durch Hilfskräfte des Studiengangs, dem Preis-Leistungs-Verhältnis, den Räumen, Informationen, der Supervision und der beruflichen Selbstreflexion. Zusätzlich zu den studienbegleitenden Seminar- und Semesterevaluationen wurden nach vollständiger Beendigung des Masterstudienganges qualitative Interviews unter Einbeziehung standardisierter Beurteilungsskalen als 
katamnestische Nacherhebung der Veränderungen des beruflichen Wissens, der persönlichen Kompetenzen und des beruflichen Status infolge des absolvierten Masterstudiums durchgeführt; zusätzlich wurden alle Masterstudierenden des ersten Kurses mittels standardisiertem Fragebogen zu den benannten Themen anonym befragt.

\section{Ergebnisse}

\section{Seminarevaluation}

Die Seminarevaluationen wurden im Anschluss an jedes Seminar erhoben und dienten vor allem der Qualitätssicherung des Lehrcurriculums und der Einzelveranstaltungen durch die unterschiedlichen Dozenten. Abb. 2 stellt die Gesamtbewertungen aller ermittelten Maße zur Qualitätssicherung hinsichtlich struktureller Bedingungen der Seminare (Transparenz, Dokumentation, Schwierigkeit und Umfang des Lehrstoffes), der beruflichen Relevanz und des fachlichen Austauschs zwischen den Teilnehmern anhand der Top Boxes (Werte 1 und 2) dar, zusammengefasst für alle durchgeführten Seminare. Dabei zeigt sich, dass annähernd drei Viertel der Studierenden des ersten Masterkurses die strukturellen Bedingungen der Seminare in hohem Maße als sehr positiv beurteilten. Auch die berufliche Relevanz und der fachliche Austausch zwischen den Teilnehmern wurde mehrheitlich positiv bewertet, weist aber für etwa $38 \%$ der Studierenden auch Verbesserungsbedarf auf. Die Gesamtbewertung der Unterrichtsmethoden, der Art und Weise der Lehrstoffvermittlung und des Auftretens der Dozenten fiel ebenfalls bei der überwiegenden Mehrheit der Studierenden positiv aus (Abb. 3).

Es ist darauf hinzuweisen, dass alle Bewertungen der Seminare in einem hoch signifikanten Zusammenhang $(p \geq 0,00)$ mit der Selbstbeurteilung der eigenen Verfassung am Seminartag stehen: Je besser das persönliche Wohlbefinden ist, desto besser sind auch die Seminarbeurteilungen hinsichtlich aller zu bewertenden Qualitätskriterien und je besser die persönliche Zufriedenheit mit den Seminaren ist, desto besser ist auch das persönliche Befinden.

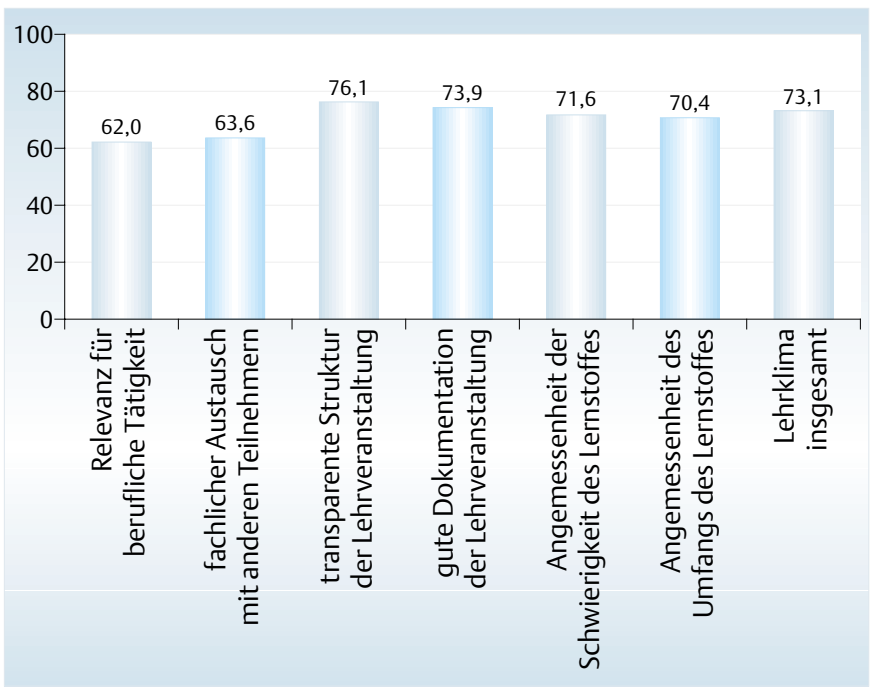

Abb. 2 Seminarevaluationen - Top Boxes in \% (Skala von „1 = trifft voll zu“ bis „6 = trifft gar nicht zu“, $\mathrm{n}=24$ Studierende; Zahl der Einzelurteile $n=1,216$ ).

\section{Semesterevaluation}

In der Semesterevaluation, die jeweils zum Ende eines jeden Semesters erhoben wurde, zeigen sich besonders positive Beurteilungen vor allem im Bereich der Supervision und der beruflichen Selbstreflexion: In der Gesamtbeurteilung bewerten 97\% die berufliche Selbstreflexion und alle Teilnehmer die Supervision als „sehr gut“ oder „gut“. In diesen Beurteilungen zeigen sich keine signifikanten Veränderungen im Verlaufe der vier Weiterbildungssemester (Tab. 2). Hinsichtlich der organisatorischen Rahmenbedingungen des Masterstudiengangs bewerten $60 \%$ der Teilnehmer des ersten Kurses die Verwaltung als besonders gut; hier zeigen sich signifikante Verbesserungen vom ersten hin zum vierten Semester (Tab. 2). Auch der Service durch die Hilfskräfte des Masterstudiengangs, die den Dozenten und Studierenden während der Gesamtdauer der Seminare unterstützend zur Verfügung stehen, wird durchgängig als sehr gut beurteilt mit einer leichten Verbesserung zum Ende des Weiterbildungsgangs.

Kritischer bewertet und daher verbesserungswürdig stellen sich vor allem die Koordination der Lehrveranstaltungen und die Organisation, aber auch die Räumlichkeiten, in denen die Seminare stattfanden, und die vorab gegebenen Informationen dar: Diese werden durchschnittlich mit Werten von 2,9 bis 3,5 beurteilt (Tab. 2), weisen also zumindest für einen größeren Anteil der Teilnehmer spezifische negative Aspekte auf. Das Preis-Leistungs-Verhältnis wird mit einem Mittelwert von 2,7 mehrheitlich und im Verlauf der Semester konstant als befriedigend bewertet.

\section{Katamnestische Nacherhebung nach Beendigung des Masterstudiums}

Die Rücklaufquote der katamnestischen Befragung mittels standardisiertem Fragebogen nach Beendigung der Masterstudienseminare betrug $54,66 \%(n=13)$. Die berufliche Position konnte während des Masterstudiums von sieben Absolventen (53,8\%) gehalten und von fünf Absolventen (38,5\%) verbessert werden; Verbesserungen der beruflichen Situation beinhalteten dabei eine Übernahme neuer Aufgaben auf der bestehenden Arbeitsstelle $(n=1)$ oder einer gleichwertigen neuen Arbeitsstelle $(n=2)$, eine höhere Bezahlung bzw. höhere tarifliche Eingruppierung $(n=1)$ und eine sicherere Positionierung im Team $(n=1)$. Verschlechterungen der beruflichen Positionierung ergaben sich während des Masterstudiums bei einem Absolventen in Form von Einschränkungen der Aufgabengebiete und einer schlechteren Bezahlung bzw. niedrigeren tariflichen Eingruppierung.

Der Masterstudiengang Suchthilfe wird von den Studenten des ersten Kurses rückblickend überwiegend positiv bewertet: Bei einer Abwägung aller positiven und negativen Aspekte im Verlaufe der zwei Studienjahre bewerten die Absolventen den Studiengang auf einer sechsstufigen Skala von „sehr gut“ bis „ungenügend" durchschnittlich mit 2,2 und 76,9\% der Masterstudenten des ersten Kurses würden sich auch aktuell wieder dafür entscheiden, dieses Studium zu absolvieren. Mehr als zwei Drittel der Absolventen gehen von deutlich besseren Chancen für ihre zukünftige berufliche Weiterentwicklung im Bereich der Suchthilfe infolge ihres Masterabschlusses aus, würden auch den Masterstudiengang Suchthilfe der Katholischen Fachhochschule NW weiterempfehlen und sehen rückblickend den zeitlichen und 


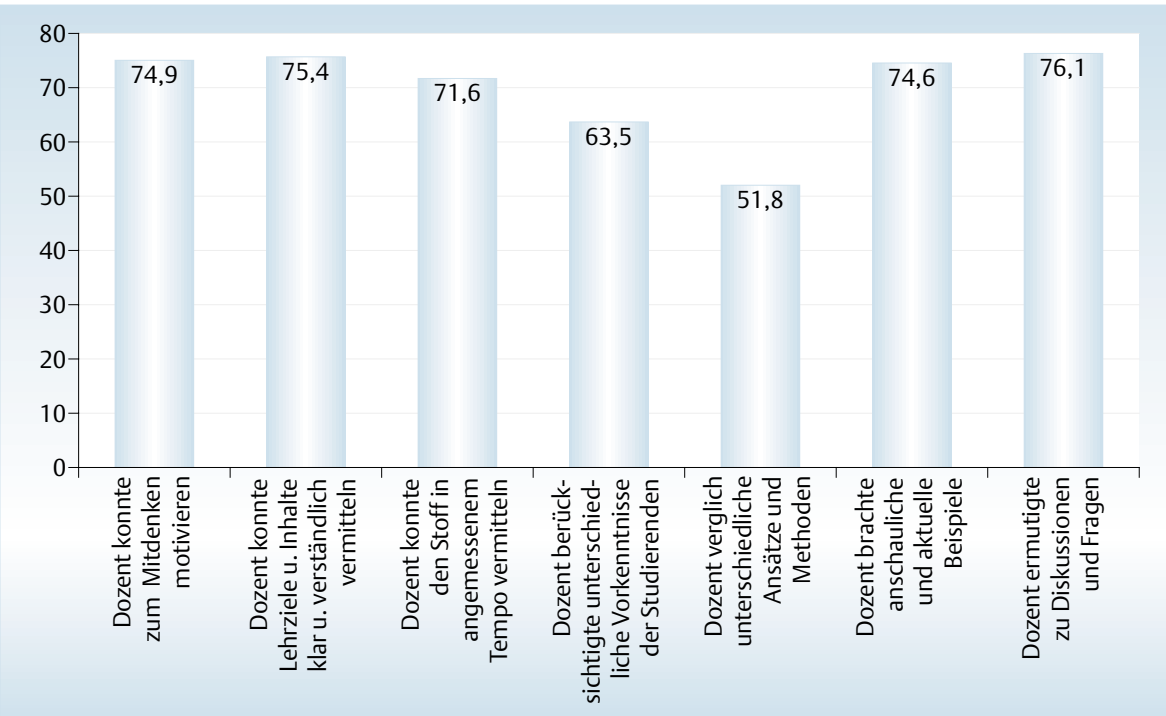

Abb. 3 Dozentenevaluationen - Top Boxes in \% (Skala von " $1=$ trifft voll zu“ bis „6 = trifft gar nicht zu“, $\mathrm{n}=24$ Studierende; Zahl der Einzelurteile $n=1,216$ ).

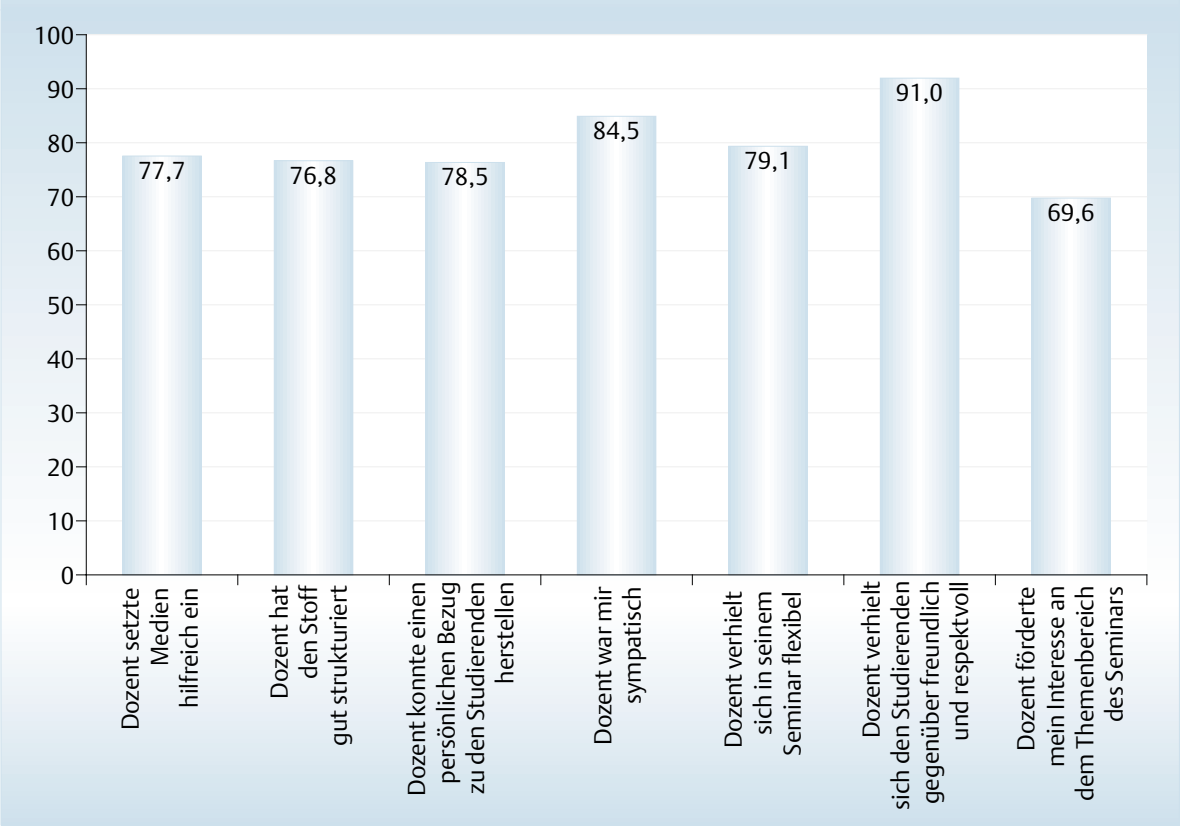

finanziellen Aufwand, den sie für die Weiterbildung eingebracht haben, als angemessen und gewinnbringend an (Tab. 3). Die auch im Rückblick überdurchschnittliche Beurteilung des Masterstudiengangs Suchthilfe gründet sich u.a. auf folgende positiv erlebte Aspekte des Studiums:

- Qualität und Inhalte der Lehrveranstaltungen: Die Zufriedenheit mit den Lehrinhalten des Studiums war mit einem Mittelwert von 4,8 insgesamt hoch; dies gilt auch für den Informationsgehalt, die Praxisrelevanz, Nutzbarkeit und praktische Umsetzung der Lehrinhalte im beruflichen Alltag hinsichtlich der vermittelten theoretischen Grundlagen, diagnostischen Fertigkeiten und therapeutischen Methoden. Besonders positiv wurde von den Absolventen wahrgenommen, dass in den Lehrveranstaltungen immer auch aktuelle Themen der Suchthilfe Berücksichtigung fanden. Verbesserungswürdig ist die Vermittlung von Qualitätssicherung und Therapiekontrolle und die Transparenz der Leistungsanforderungen im Studium, z.B. hinsichtlich Bewertungen von Leistungsnachweisen (Tab. 4).
- Zusammenarbeit mit den Dozenten des Masterstudiengangs: Aus Absolventensicht ist eine hohe Qualität der Lehre durch die Dozenten seit Einführung des Masterstudiums konstant gegeben: Mit der inhaltlichen Gestaltung der Lehrgebiete durch die Dozenten waren 15,4\% der Absolventen sehr zufrieden, 84,6\% zufrieden; mit der beruflichen Qualifikation und Erfahrung der Dozenten 38,5\% sehr zufrieden, 61,5\% zufrieden und mit der didaktischen Kompetenz 7,7\% sehr zufrieden, $69,2 \%$ zufrieden und 23,1\% weniger zufrieden.

- Zusammenarbeit der Kommilitonen untereinander: Die Zusammenarbeit zwischen den Kommilitonen des ersten Kurses war auch in deren Selbsteinschätzung von einem hohen fachlichen Austausch, gegenseitigen Lernerfahrungen und einer durch die Supervisoren unterstützten positiven Auseinandersetzung und Konfliktbewältigung geprägt; die Arbeitsatmosphäre untereinander zeichnete sich wenig durch Konkurrenzdenken, sondern vielmehr durch gegenseitige Unterstützung im Lernprozess aus (Tab. 4). 
Tab. 2 Semesterevaluation - Mittelwertvergleich (Werte 1 und 2 auf einer Skala von „1 = sehr gut“ bis „6 = sehr schlecht“, Angabe der Top Boxes in Klammern; $\mathrm{n}=88$ )

\begin{tabular}{|c|c|c|c|c|c|c|}
\hline & \multirow[t]{2}{*}{ gesamt } & \multirow[b]{2}{*}{$\begin{array}{l}I \\
(n=19)\end{array}$} & \multicolumn{3}{|c|}{ Semester } & \multirow[t]{2}{*}{ sig. } \\
\hline & & & $\begin{array}{l}\text { II } \\
(n=16)\end{array}$ & $\begin{array}{l}\text { III } \\
(n=21)\end{array}$ & $\begin{array}{l}\text { IV } \\
(n=12)\end{array}$ & \\
\hline Koordination der Lehrangebote & $\begin{array}{l}3,2 \\
(24 \%)\end{array}$ & $\begin{array}{l}3,5 \\
(16 \%)\end{array}$ & $\begin{array}{l}3,1 \\
(25 \%)\end{array}$ & $\begin{array}{l}3,4 \\
(14 \%)\end{array}$ & $\begin{array}{l}2,6 \\
(50 \%)\end{array}$ & 0,04 \\
\hline Verwaltung & $\begin{array}{l}2,4 \\
(60 \%)\end{array}$ & $\begin{array}{l}2,8 \\
(32 \%)\end{array}$ & $\begin{array}{l}2,5 \\
(56 \%)\end{array}$ & $\begin{array}{l}2,1 \\
(57 \%)\end{array}$ & $\begin{array}{l}2,1 \\
(75 \%)\end{array}$ & 0,00 \\
\hline Organisation & $\begin{array}{l}3,0 \\
(27 \%)\end{array}$ & $\begin{array}{l}3,0 \\
(32 \%)\end{array}$ & $\begin{array}{l}3,1 \\
(31 \%)\end{array}$ & $\begin{array}{l}3,2 \\
(10 \%)\end{array}$ & $\begin{array}{l}2,7 \\
(42 \%)\end{array}$ & n.s. \\
\hline Service (Hiwis) & $\begin{array}{l}1,3 \\
(98 \%)\end{array}$ & $\begin{array}{l}1,6 \\
(95 \%)\end{array}$ & $\begin{array}{l}1,3 \\
(100 \%)\end{array}$ & $\begin{array}{l}1,3 \\
(100 \%)\end{array}$ & $\begin{array}{l}1,1 \\
(100 \%)\end{array}$ & 0,05 \\
\hline Preis-Leistungs-Verhältnis & $\begin{array}{l}2,7 \\
(38 \%)\end{array}$ & $\begin{array}{l}2,7 \\
(42 \%)\end{array}$ & $\begin{array}{l}2,7 \\
(44 \%)\end{array}$ & $\begin{array}{l}2,8 \\
(38 \%)\end{array}$ & $\begin{array}{l}2,8 \\
(25 \%)\end{array}$ & n. s. \\
\hline Räume & $\begin{array}{l}3,5 \\
(16 \%)\end{array}$ & $\begin{array}{l}3,7 \\
(16 \%)\end{array}$ & $\begin{array}{l}3,4 \\
(19 \%)\end{array}$ & $\begin{array}{l}3,4 \\
(19 \%)\end{array}$ & $\begin{array}{l}3,8 \\
(8 \%)\end{array}$ & n.s. \\
\hline Informationen & $\begin{array}{l}2,9 \\
(37 \%)\end{array}$ & $\begin{array}{l}2,7 \\
(37 \%)\end{array}$ & $\begin{array}{l}3,0 \\
(31 \%)\end{array}$ & $\begin{array}{l}3,0 \\
(38 \%)\end{array}$ & $\begin{array}{l}2,8 \\
(42 \%)\end{array}$ & n. s. \\
\hline Supervision & $\begin{array}{l}1,4 \\
(100 \%)\end{array}$ & $\begin{array}{l}1,4 \\
(100 \%)\end{array}$ & $\begin{array}{l}1,4 \\
(100 \%)\end{array}$ & $\begin{array}{l}1,5 \\
(100 \%)\end{array}$ & $\begin{array}{l}1,1 \\
(100 \%)\end{array}$ & n. s. \\
\hline berufliche Selbstreflexion & $\begin{array}{l}1,4 \\
(97 \%)\end{array}$ & $\begin{array}{l}1,5 \\
(95 \%)\end{array}$ & $\begin{array}{l}1,6 \\
(94 \%)\end{array}$ & $\begin{array}{l}1,4 \\
(100 \%)\end{array}$ & $\begin{array}{l}1,1 \\
(100 \%)\end{array}$ & n. s. \\
\hline
\end{tabular}

Tab. 3 Katamnestische Gesamtbewertung des Masterstudiums (Skala von „1= trifft nicht zu“ bis „6= trifft zu“, Top Boxes= Werte 5 und 6; $\mathrm{n}=13$ )

\begin{tabular}{|c|c|c|c|c|c|}
\hline Alles in allem ... & Top Boxes & Mittelwert & Min. & Max. & $S D$ \\
\hline finde ich, dass sich der zeitliche Aufwand für die Weiterbildung für mich gelohnt hat. & $69,3 \%$ & 4,8 & 3 & 6 & 0,8 \\
\hline $\begin{array}{l}\text { rechne ich mit deutlich besseren Chancen für neue Stellenbewerbungen oder eine } \\
\text { Verbesserung meiner beruflichen Position durch die Weiterbildung. }\end{array}$ & $69,3 \%$ & 4,8 & 2 & 6 & 1,2 \\
\hline würde ich das Masterstudium Suchthilfe der KFH NW auch anderen weiterempfehlen. & $69,3 \%$ & 4,8 & 3 & 6 & 1,0 \\
\hline finde ich, dass sich der finanzielle Aufwand für die Weiterbildung für mich gelohnt hat. & $53,9 \%$ & 4,5 & 2 & 6 & 1,1 \\
\hline $\begin{array}{l}\text { stehen Aufwand und Nutzen der Weiterbildung in einem angemessenen Verhältnis } \\
\text { zueinander. }\end{array}$ & $53,8 \%$ & 4,3 & 3 & 5 & 0,9 \\
\hline wird das Masterstudium Suchthilfe in meinem beruflichen Umfeld positiv bewertet. & $46,2 \%$ & 4,4 & 2 & 6 & 1,1 \\
\hline
\end{tabular}

- Kompetenzentwicklung und Selbsterfahrung: Die Entwicklung der beruflichen und persönlichen Kompetenzen war aus Sicht der Absolventen in einem ausgeprägten Maße durch die Reflexion des eigenen Erlebens und Verhaltens und eine steigende Sicherheit im Umgang mit Klienten, vor allem Patienten mit Suchterkrankungen bestimmt. Durch die Weiterbildung konnten Fähigkeiten und Fertigkeiten erworben werden, die im Selbsterleben eine weitere professionelle Arbeit in der Suchthilfe gewährleisten und die in der praktischen Arbeit bereits während des Studiums genutzt werden konnten. Nach den zwei Weiterbildungsjahren identifizieren sich 61,6\% der Teilnehmer mit der Bezeichnung „Suchttherapeut“ und 38,5\% mit einer verhaltenstherapeutisch orientierten Arbeitsweise. Lernerfahrungen im Studium werden zu 69,2\% auch als unterstützend für die persönliche Entwicklung beurteilt, obwohl fast zwei Drittel der Absolventen höhere Belastungen des Privatlebens durch den zeitlichen und/oder finanziellen Aufwand der Weiterbildung erlebten (Tab.4).

\section{Schlussfolgerungen}

Der postgraduale Masterstudiengang „Master of Science (M. Sc.) in Addiction Prevention and Treatment" weist aus Sicht der Absolventen bereits in der ersten Weiterbildungsgruppe eine hohe Qualität hinsichtlich der theoretischen und praktischen therapeutischen Ausbildung auf. Die befragten Absolventen berichten - trotz bereits zum Teil vorhandener therapeutischer Ausbildungen und langjähriger Berufserfahrungen in der Suchthilfe - von einer deutlichen Professionalisierung, Kompetenzsteigerung und Reflexion ihrer Arbeit mit Patienten mit Suchterkrankungen; dies spiegelt sich dann auch in einer Verbesserung der beruflichen Position bei 38,5\% der Teilnehmer während der Weiterbildung wider. Verbesserungsbedarf des Masterstudiums ergibt sich zukünftig vor allem hinsichtlich der Vermittlung von Qualitätssicherung in der Therapie und hinsichtlich des organisatorischen Aufbaus und der Transparenz von Lehrveranstaltungen und Leistungsanforderungen. 


\begin{tabular}{|c|c|c|c|c|c|}
\hline & TopBoxes & Mittelwert & Min. & Max. & $S D$ \\
\hline \multicolumn{6}{|l|}{ Qualität und Inhalte der Lehrveranstaltungen: „Im Nachhinein betrachtet ...“ } \\
\hline $\begin{array}{l}\text { wurden in den Lehrveranstaltungen auch immer wieder aktuelle Themen der Suchthilfe } \\
\text { berücksichtigt. }\end{array}$ & $84,6 \%$ & 5,0 & 3 & 6 & 0,8 \\
\hline $\begin{array}{l}\text { kann ich die gelernten Theorien, z. B. zur Suchttherapie, zu Ursachen von Sucht usw. auch } \\
\text { gut in meinem beruflichen Alltag umsetzen und nutzen. }\end{array}$ & $76,9 \%$ & 4,7 & 1 & 6 & 1,3 \\
\hline war ich mit den Lehrinhalten der Veranstaltungen alles in allem zufrieden. & $69,2 \%$ & 4,8 & 4 & 6 & 0,6 \\
\hline war der Informationsgehalt der theoretischen Lehrveranstaltungen hoch & $69,2 \%$ & 4,8 & 3 & 6 & 0,6 \\
\hline wurden die theoretischen Grundlagen der praktischen Übungen gut vermittelt. & $69,2 \%$ & 4,8 & 3 & 6 & 0,8 \\
\hline $\begin{array}{l}\text { kann ich die gelernten diagnostischen Erkenntnisse und Fähigkeiten in meinem } \\
\text { beruflichen Alltag gut umsetzen und nutzen. }\end{array}$ & $69,2 \%$ & 4,5 & 1 & 6 & 1,2 \\
\hline wurde in den Lehrveranstaltungen viel praxisrelevantes Wissen vermittelt. & $53,9 \%$ & 4,7 & 4 & 6 & 0,8 \\
\hline $\begin{array}{l}\text { wurde in den Praxisübungen und Lehrveranstaltungen auch immer genügend Bezug } \\
\text { genommen auf das Arbeitsfeld Suchthilfe. }\end{array}$ & $53,9 \%$ & 4,6 & 4 & 6 & 0,7 \\
\hline $\begin{array}{l}\text { kann ich die gelernten therapeutischen Methoden und Fähigkeiten in meinem beruflichen } \\
\text { Alltag gut umsetzen und nutzen. }\end{array}$ & $46,2 \%$ & 4,2 & 2 & 6 & 1,1 \\
\hline $\begin{array}{l}\text { wurde in den Lehrveranstaltungen hinreichend auf ethische und berufsständige Fragen } \\
\text { eingegangen. }\end{array}$ & $46,2 \%$ & 4,2 & 2 & 5 & 0,9 \\
\hline habe ich auch einiges über Qualitätssicherung und Therapiekontrolle gelernt. & $30,8 \%$ & 3,9 & 2 & 5 & 1,0 \\
\hline $\begin{array}{l}\text { war für mich in der Regel ersichtlich, welche Anforderungen in einem Fach, z. B. bei den } \\
\text { Leistungsnachweisen gestellt wurden. }\end{array}$ & $23,1 \%$ & 3,3 & 1 & 5 & 1,5 \\
\hline \multicolumn{6}{|l|}{ Qualität der Zusammenarbeit mit Kommilitonen: „Im Nachhinein betrachtet ...“ } \\
\hline fand viel Austausch über berufliche Erfahrungen und Arbeitsweisen statt. & $69,3 \%$ & 4,8 & 3 & 6 & 1,0 \\
\hline $\begin{array}{l}\text { haben Supervisoren uns auch gut darin unterstützt, mit Konflikten in der Gruppe zurecht- } \\
\text { zukommen. }\end{array}$ & $69,3 \%$ & 4,8 & 3 & 6 & 1,2 \\
\hline $\begin{array}{l}\text { war das Arbeitsklima zwischen den Studierenden so, dass man auch gut miteinander } \\
\text { lernen konnte. }\end{array}$ & $69,3 \%$ & 4,6 & 2 & 6 & 1,0 \\
\hline gab es einige Kommilitonen, von deren Wissen und Erfahrungen ich viel lernen konnte. & $61,4 \%$ & 4,8 & 3 & 6 & 1,1 \\
\hline haben wir viel miteinander gelacht und Spaß gehabt. & $46,2 \%$ & 4,5 & 3 & 6 & 1,0 \\
\hline $\begin{array}{l}\text { gab es doch schon ziemlich viele harte, nicht gerade freundliche Diskussionen zwischen } \\
\text { einzelnen Studierenden. }\end{array}$ & $23,1 \%$ & 2,9 & 1 & 6 & 1,6 \\
\hline $\begin{array}{l}\text { war die Stimmung zwischenzeitlich so schlecht, dass ich gar keine Lust mehr hatte, zu } \\
\text { den Lehrveranstaltungen zu kommen. }\end{array}$ & $23,1 \%$ & 2,5 & 1 & 6 & 2,0 \\
\hline gab es ziemlich viel Konkurrenz um die Frage, wer der „bessere Suchttherapeut“ ist. & $15,4 \%$ & 3,4 & 1 & 6 & 1,5 \\
\hline \multicolumn{6}{|l|}{ Persönliche Kompetenzentwicklung und Selbsterfahrung } \\
\hline $\begin{array}{l}\text { Ich habe innerhalb des Studiums verstärkt mein persönliches Erleben und Verhalten im } \\
\text { Umgang mit Klienten reflektiert. }\end{array}$ & $100,0 \%$ & 5,2 & 5 & 6 & 0,4 \\
\hline $\begin{array}{l}\text { Durch das Masterstudium habe ich vor allem auch Sicherheit im Umgang mit schwierigen } \\
\text { Therapiesituationen gewonnen. }\end{array}$ & $84,6 \%$ & 5,0 & 3 & 6 & 0,8 \\
\hline $\begin{array}{l}\text { Durch das Masterstudium habe ich noch mehr Sicherheit im Umgang mit Suchtpatienten } \\
\text { gewonnen. }\end{array}$ & $76,9 \%$ & 5,2 & 4 & 6 & 0,8 \\
\hline $\begin{array}{l}\text { Ich fühle mich durch die Weiterbildung gut ausgebildet, um im Bereich Suchthilfe } \\
\text { professionell arbeiten zu können. }\end{array}$ & $76,9 \%$ & 4,5 & 1 & 6 & 1,3 \\
\hline $\begin{array}{l}\text { Insgesamt konnte ich einige Lernerfahrungen auch für meine persönliche Entwicklung gut } \\
\text { nutzen. }\end{array}$ & $69,2 \%$ & 4,8 & 3 & 6 & 0,8 \\
\hline $\begin{array}{l}\text { Ich habe die Weiterbildung auch dafür nutzen können, mir selbst über meine Haltungen } \\
\text { und Einstellungen als Therapeut klarer zu werden. }\end{array}$ & $61,6 \%$ & 4,7 & 3 & 6 & 0,9 \\
\hline $\begin{array}{l}\text { Ich habe in der Weiterbildung einiges über meine Fähigkeiten und fachliche Ressourcen } \\
\text { als Therapeut/in gelernt. }\end{array}$ & $61,6 \%$ & 4,6 & 3 & 6 & 1,0 \\
\hline $\begin{array}{l}\text { Das Masterstudium hat durch den zeitlichen und/oder finanziellen Aufwand mein } \\
\text { Privatleben sehr belastet. }\end{array}$ & $61,6 \%$ & 4,5 & 2 & 6 & 1,6 \\
\hline $\begin{array}{l}\text { Ich konnte die Weiterbildung gut nutzen für meine eigene praktisch-therapeutische Arbeit } \\
\text { in der Suchthilfe. }\end{array}$ & $61,6 \%$ & 4,5 & 1 & 6 & 1,3 \\
\hline Alles in allem fühle ich mich durch die Weiterbildung jetzt auch wirklich als „Suchttherapeut“. & $61,6 \%$ & 4,5 & 1 & 6 & 1,6 \\
\hline $\begin{array}{l}\text { Ich fühle mich jetzt auch als „richtiger Therapeut“, der kompetent und fundiert mit Klienten } \\
\text { therapeutisch arbeiten kann. }\end{array}$ & $38,5 \%$ & 4,2 & 1 & 6 & 1,4 \\
\hline Ich bin seit der Weiterbildung alles in allem zufriedener mit meiner beruflichen Situation. & $38,5 \%$ & 4,1 & 1 & 6 & 1,3 \\
\hline Ich arbeite seit der Weiterbildung deutlich stärker verhaltenstherapeutisch. & $38,5 \%$ & 4,1 & 1 & 6 & 1,4 \\
\hline $\begin{array}{l}\text { Meine Beziehungen zu Partner/in, Familie und/oder Freunden sind durch das Masterstudium } \\
\text { nicht beeinträchtigt worden. }\end{array}$ & $38,5 \%$ & 3,5 & 1 & 6 & 1,9 \\
\hline Ich bin seit der Weiterbildung alles in allem zufriedener mit meiner privaten Situation. & $15,4 \%$ & 2,9 & 1 & 6 & 1,7 \\
\hline $\begin{array}{l}\text { Ehrlich gesagt habe ich das Gefühl, mir fehlt auch nach der Weiterbildung die Kompetenz, } \\
\text { als Therapeut eigenständig und selbstverantwortlich zu arbeiten. }\end{array}$ & $7,7 \%$ & 1,9 & 1 & 6 & 1,5 \\
\hline $\begin{array}{l}\text { Im Masterstudium habe ich mich auch persönlich verändert, was mein Privatleben eher } \\
\text { belastet hat. }\end{array}$ & - & 1,8 & 1 & 3 & 0,8 \\
\hline
\end{tabular}

